\title{
The New Surgical Instrument in Peritoneal Endometriosis Resection
}

\author{
Garri Tchartchian $^{1 *}$, Andreas Hackethal ${ }^{2}$, Bernd Bojahr ${ }^{1}$, Rudy Leon De Wilde ${ }^{3}$ \\ ${ }^{1}$ Klinik für Minimal Invasive Chirurgie, Berlin, Germany \\ ${ }^{2}$ Zentrum für Frauenheilkunde und Geburtshilfe, Universitätsklinikum Gießen und Marburg GmbH, Giessen, Germany \\ ${ }^{3}$ Pius-Hospital, Klinik für Frauenheilkunde, Geburtshilfe und Gynäkologische Onkologie, Oldenburg, Germany \\ Email: *g.tchartchian@mic-berlin.de
}

Received May 25, 2012; revised June 24, 2012; accepted July 6, 2012

\begin{abstract}
Purpose: Peritoneal resection for the therapy of oncological diseases as well as endometriosis is associated with major problems as injury to neighboring organs and difficulties in the establishment of the necessary hemostasis. In this paper we present a new dissection tool, the "Jet Grasper" (KARL STORZ ${ }^{\circledR}$ ), which represents a further step towards simple, safe and effective laparoscopic peritoneal resection. Methods: The "Jet Grasper", was developed to enable selective dissection of the peritoneum and, if necessary, other organs in the case of adhesions. For the tissue resection different techniques can be applied (e.g. ultrasound, mechanical or electrosurgical energy). Results: The tool features a dissection forceps with a narrow slit at the end of the jaw. Further selective dissection is achieved by applying water pressure retroperitoneally via a controlled water pressure pump. Furthermore a diameter difference between the jaw and the sheath potentiates dissection. The thin jaws are introduced precisely to separate and/or dissect layers in a physiological manner to enable further dissection of the peritoneum at a safe distance. Larger vessels can subsequently be targeted for bipolar coagulation in the opened retroperitoneal area. As we are exclusively presenting a completely new device, no patient data are included here. However, a prospective randomized study already started in April 2012, and will be reported after completion in two years. Conlusion: The new dissection tool combines selective dissection and resection under controlled water pressure, known from aquadissection. It provides enhanced and atraumatic possibilities in performing pelvic peritoneal resection via selective dissection and reduction of blood loss.
\end{abstract}

Keywords: Jet Grasper; Peritoneal Resection; Complication; Reduction; Aquadissection; Laparoscopic Surgery; Endometriosis

\section{Introduction}

The peritoneum is an organ with multiple functions which is involved in various pathogenetic processes. Oncological diseases as well as endometriosis are gynecopathological conditions which partially or fully affect the peritoneum $[1,2]$. Peritoneal resection, either open or minimally-invasive, is one of the therapy methods used to treat these medical conditions. In endometriosis surgery, peritoneal resection is understood as the layer-specific dissection of the peritoneum from the underlying tissue and organs with subsequent resection under protection of the neighboring organs.

There are two problems associated with this surgical method: to perform resection without causing injury to neighboring organs and to establish the necessary hemostasis [3]. Any complications arising during selective dissection make hemostasis and further dissection difficult, resulting in possible organ complications. The use of a new

${ }^{*}$ Corresponding author. dissection tool, therefore, represents a further step towards simple, safe and effective peritoneal resection.

\section{Materials and Methods}

The aim of this therapy is the minimally invasive pelvic peritoneal resection of the affected areas. A new dissecttion tool, the "Jet Grasper" (KARL STORZ ${ }^{\circledR}$ ), was developed for this purpose. This dissecting forceps enables selective dissection of the peritoneum and, if necessary, other organs in the case of adhesions [4] (see images). For the tissue resection different techniques can be applied (e.g. ultrasound, mechanical or electrosurgical energy).

\section{Results}

The tool features a dissection forceps (Figures 1 and 2) with a narrow slit at the end of the jaw. Once dissection (Figure 3) of the peritoneum starts, further selective dissection is achieved by applying water under pressure 
into the retroperitoneum via a controlled water pressure pump (HAMOU-ENDOMAT ${ }^{\circledR}$, KARL STORZ ${ }^{\circledR}$ ). Furthermore a diameter difference between the jaw and the sheath potentiates dissection. The thin jaws are introduced precisely to separate and/or dissect layers in a physiological manner. A bed of water is thus created for at least 5 minutes between the peritoneum and an organ, i.e. pelvic wall. This artificial dislodgement enables further dissection of the peritoneum at a safe distance, i.e. to the uterus, ureter, nerves and pelvic vessels, whereby smaller retroperitoneal vessels are displaced and preserved. Larger vessels can subsequently be targeted for bipolar coagulation in the opened retroperitoneal area: this solves the second major problem associated with conventional peritoneal resection.
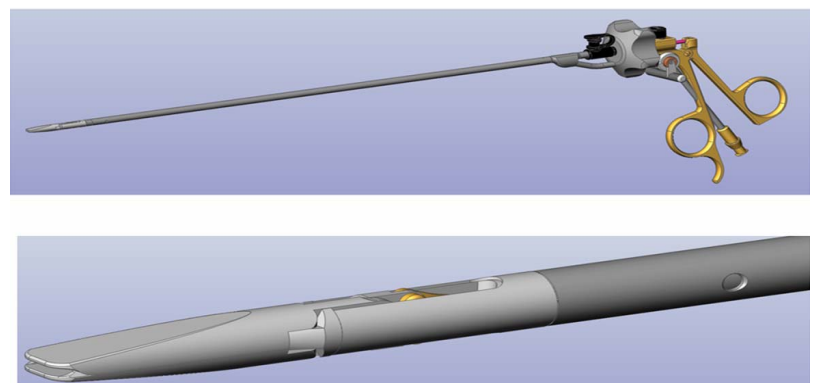

Figure 1. Tool feature 1: dissection forceps.

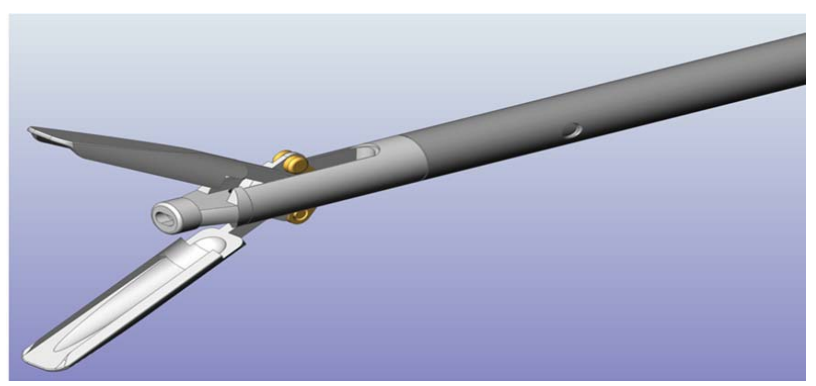

Figure 2. Tool feature 1: dissection forceps.

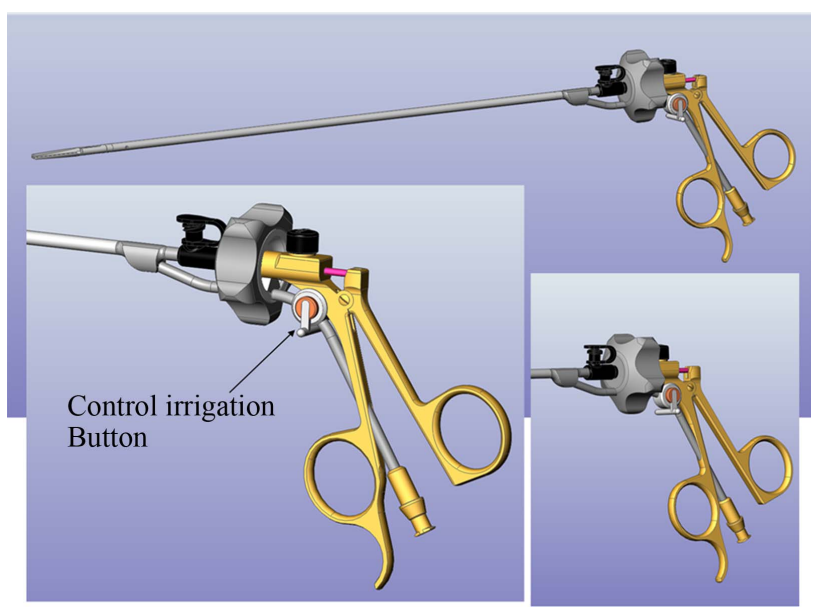

Figure 3. Tool feature 2: water pressure pump.

\section{Discussion}

Aquadissection is a well-known technique [5]. The new tool combines two proven operating principles: selective dissection under controlled water pressure and ablation [6]. Water pressure can be adjusted according to the aim of surgery (peritoneal resection, bowel adhesiolysis and other preparation).

Utilizing a dissection forceps enables preservation of tissue layers and organs and simplifies resection of the peritoneum considerably. Furthermore, the exposed retroperitoneal vessels can be directly hemostatically treated. Surgery can be performed at a safe distance to the uterus and the pelvic vessels. Potential problems may arise if there is a firm connection between the peritoneum and the organ, i.e. in the case of scarring or adherence towards the rectum. Here, a lower water pressure should be applied to prevent injuries.

As a result of a gentle build-up in water pressure in the retroperitoneal space, the fine dissection properties of the tool, and immediate subsequent resection, fluid loss is only at about $10 \%$. The fluid is drained with a suction device after resection. In all of the current cases, a maximum of $2000 \mathrm{ml}$ lactated Ringer's solution was used, the maximum loss of fluid amounting to about $200 \mathrm{ml}$.

As the dissection forceps is a tool that introduces fluids, possible resorption should be taken into account. Using a $\mathrm{NaCl}$ solution keeps the risk of a "fluid-overload syndrome" to a minimum [7]. Other fluids require intraoperative fluid management and postoperative monitoring.

As we are exclusively presenting a completely new device, no patient data are included here. However, a prospective randomized study already started in April 2012, and will be reported after completion in two years.

\section{Conclusion}

The new dissection tool provides enhanced and atraumatic possibilities in performing pelvic peritoneal resection with selective dissection and reduction of blood loss. The use of the instrument for other surgical interventions (such as bowel adhesiolysis) is possible. Comparative studies are currently being carried out to evaluate the safety and efficiency of this method.

\section{Acknowledgements}

This study was sponsored by Karl Storz ${ }^{\circledR}$.

\section{REFERENCES}

[1] I. Brosens and G. Benagiano, "Endometriosis, a Modern Syndrome," Indian Journal of Medical Research, Vol. 133, No. 6, 2011, pp. 581-593.

[2] J. K. Slack-Davis, K. A. Atkins, C. Harrer, E. D. Hershey and M. Conaway, "Vascular Cell Adhesion Molecule-1 Is 
a Regulator of Ovarian Cancer Peritoneal Metastasis,” Cancer Research, Vol. 69, 2009, pp. 1469-1476. doi:10.1158/0008-5472.CAN-08-2678

[3] J. Cooper, R. Gimpelson, P. Laberge, D. Galen, J. G. Garza-Leal, J. Scott, N. Leyland, P. Martyn and J. Liu, “A Randomized, Multicenter Trial of Safety and Efficacy of the NovaSure System in the Treatment of Menorrhagia," The Journal of the American Association of Gynecologic Laparoscopists, Vol. 9, No. 4, 2002, pp. 418-428. doi:10.1016/S1074-3804(05)60513-0

[4] R. L. De Wilde, G. Trew and Expert Adhesions Working Party of the European Society of Gynecological Endoscopy (ESGE), "Postoperative Abdominal Adhesions and
Their Prevention in Gynaecological Surgery. Expert Consensus Position,” Gynecological Surgery, Vol. 4, No. 3, 2007, pp. 161-168.

[5] P. B. Marshburn and J. F. Hulka, "A Simple IrrigatorAspirator Cannula for Laparoscopy: The Stewart System," Obstetrics \& Gynecology, Vol. 75, No. 3, 1990, pp. 458460.

[6] M. C. Merell, "Laparoscopic Surgery: A Colloquium," Springer, New York, 1999.

[7] A. Lethaby, S. Shepperd, I. Cooke and C. Farquhar, "Endometrial Resection and Ablation versus Hysterectomy for Heavy Menstrual Bleeding,” Cochrane Database Systematic Reviews, No. 2, 1999, Article ID: CD000329. 